\title{
PREVALENCE OF NONALCOHOLIC - FATTY LIVER DISEASE IN METABOLIC SYNDROME - A HOSPITAL-BASED CROSS-SECTIONAL STUDY
}

\author{
DIVYA KAJARIA \\ Department of Kayachikitsa, All India Institute of Ayurveda, New Delhi, India. Email: divyakajaria@gmail.com
}

Received: 23 May 2017, Revised and Accepted: 20 July 2017

ABSTRACT

Objective: $21^{\text {st }}$ century is dedicated to noncommunicable diseases; it may be diabetes, hypertension, obesity, or constellation of all known as metabolic syndrome. The main objective of this paper is to assess the prevalence of nonalcoholic fatty liver disease (NFLDS) in metabolic syndrome and its impact on the severity of metabolic syndrome in term of dyslipidemia, mean systolic pressure and body mass index (BMI).

Methods: A cross-sectional study was conducted on 100 patients selected randomly during the period of January 2017 -March 2017 from the outdoor patient department of Kayachikitsa of All India Institute of Ayurveda. Patient included on the basis of a screening pro forma based on the diagnostic criteria of metabolic syndrome on the basis of Third Adult Treatment Panel of the National Cholesterol Education Program criteria and 50 controls were included in the study. Pro forma also included general information regarding previous illness, socioeconomical status, BMI, evaluation of vital parameters, and lipid and thyroid profile, along with other routine laboratory parameters. Ultrasonography report is taken as the evidence for the diagnosis of fatty liver disease.

Results: Frequency distributions and Chi-square statistics were used for categorical variables. Logistic regression analyses Students' t-test, Chi-square test, linear regression, and multiple logistic regression models were used for statistical analysis. p<0.05 was considered significant. BMI, waist circumference, mean systolic pressure, diastolic blood pressure, fasting blood sugar, triglycerides, total cholesterol, low-density lipoprotein (LDL) cholesterol were significantly higher, and high-density lipoprotein cholesterol, was significantly low in the study group compared to the control group. In the metabolic syndrome group, 67 patients (67\%) had fatty liver Grade I, 34 had Grade II fatty liver (34\%), 6 patients were having hepatomegaly with coarse echotexture $(6 \%)$ and 4 patients were having cholelithiasis. NFLDS was significantly associated with metabolic syndrome group $(\mathrm{p}<0.0001)$. There was a significant linear association between fatty infiltration in liver and BMI, total cholesterol, triglycerides, and LDL cholesterol in the metabolic syndrome group in the linear regression model. Multiple logistic regression analysis recognized the association between fatty liver disease and BMI with dyslipidemia $(\mathrm{p}<0.001)$ in the metabolic syndrome group.

Conclusion: It is concluded from this study that there is a significant association between NFLDS and metabolic syndrome, and it highlights the importance of evaluating liver profile in patients with metabolic syndrome.

Keywords: Hepatic steatosis, Dyslipidemia, Insulin resistance, Body mass index.

(C) 2017 The Authors. Published by Innovare Academic Sciences Pvt Ltd. This is an open access article under the CC BY license (http://creativecommons. org/licenses/by/4. 0/) DOI: http://dx.doi.org/10.22159/ajpcr.2017.v10i10.20159

\section{INTRODUCTION}

Metabolic syndrome as per definition is a collection of medical conditions including high blood pressure, high fasting blood sugar level, increase waist circumference, abnormal cholesterol, or triglyceride level [1]. However, in this scenario, it seems that this definition should be redefine as in practice metabolic syndrome is found to invariably associated with nonalcoholic fatty liver disease (NFLDS). Thus, to quest the prevalence of NFLDS in metabolic syndrome following clinical study is planned. NFLDS is define as accumulation of fat (steatosis) in the liver which is not caused by alcohol consumption [2]. Incidence of NAFLD is increasing in both adult and children population $[3,4]$. The exact etiology of hepatic steatosis is not well understood, through its high occurrence in obesity (about $60 \%$ of obese and $90 \%$ of morbidly obese adults are having NAFLD) [5] signifies that dietary habits, sedentary life style and to some extent hereditary factors may have determinant role in pathogenesis. NAFLD is a broad term including simple steatosis, nonalcoholic steatohepatitis (NASH), fibrosis, and rarely, progression to cirrhosis [6]. Vivid cassette between insulin resistance, oxidative stress resulting in lipid peroxidation, proinflammatory cytokines, adipokines and mitochondrial dysfunction causes NAFLD [7]. Few previous studies reported the association of NAFLD and metabolic syndrome, but the data provided are mainly epidemiological and conceptual [8-10]. Hence, there is need of a research study to validate as well as assess the extent of association ship between metabolic syndrome and NAFLD.

\section{METHODS}

This cross-sectional study was conducted in Outpatient Department of Kayachikitsa, All India Institute of Ayurveda at New Delhi. 100 known case of metabolic syndrome diagnosed on the basis of National Cholesterol Education Program-Adult Treatment Panel criteria (three out of five criteria should be positive, viz., blood pressure $\geq 130 / 85 \mathrm{mmHg}$ or taking antihypertensive medications, fasting blood glucose $>100 \mathrm{mg} / \mathrm{dl}$ or taking antidiabetic medications, triglycerides $>150 \mathrm{mg} / \mathrm{dl}$, high-density lipoprotein cholesterol [HDL-C] $<40 \mathrm{mg} / \mathrm{dl}$ in males, and $<50 \mathrm{mg} / \mathrm{dl}$ in females, waist circumference $>102 \mathrm{~cm}$ in men and $88 \mathrm{~cm}$ in women) were taken in the study group and 50 patients having no features of metabolic syndrome ( 0 out of 5 criteria) were taken in the control group. A written informed consent was obtained in the local language from every patient (or) his reliable caretaker before enrollment in the trial.

\section{Evaluating scale}

\section{Screening pro forma}

A pro forma is made for screening patients of metabolic syndrome and also assessing their demographic profile, history of previous illness and medication, anthropometric measurement including weight and height for calculating body mass index (BMI = weight in $\mathrm{Kg} /$ height in $\mathrm{m}^{2}$ ), waist circumference, measuring blood pressure 
using standard sphygmomanometers. The value of variables is taken as per standard definition, say for example BMI $<18.5 \mathrm{~kg} / \mathrm{m}$ was categorize as underweight, $\mathrm{BMI}=18.5-24.9 \mathrm{~kg} / \mathrm{m}^{2}$ as normal, overweight if BMI $=25-29.9 \mathrm{~kg} / \mathrm{m}^{2}$, and obesity if BMI $30 \mathrm{~kg} / \mathrm{m}^{2}$. Similarly, hypertension and hyperglycemia were categorized according to standard definition $[11,12]$. For measuring waist circumference, the distance between anterior superior iliac spines and the lower costal margin at the narrowest part of the waistline was calculated during expiration while putting patient in standing position.

\section{Exclusion criteria}

All patients having chronic renal disorder, congestive cardiac failure, altered thyroid profile, taking statins, or other medication that can alter the lipid profile, having past history of tuberculosis (ATT causes liver function derangement and may act as confounder) or hepatitis and history of any chronic illness such as polycystic ovarian disease and rheumatoid arthritis are also excluded keeping sick euthyroid syndrome in consideration.

\section{Collection of data}

All patients were undergone USG investigation for evaluating histopathology of the liver. Fasting blood samples were obtained for measuring FBS (venous blood samples taken after overnight fast of a minimum of $8 \mathrm{hrs}$ ); total cholesterol, low-density lipoprotein cholesterol (LDL-C), HDL-C, and triglyceride levels were measured.

\section{Statistical analysis}

Mean \pm SD and percentage were used for assessing baseline characteristics of the study participants. For analyzing difference in baseline characteristic between the study and control groups, Student's t-test was used. Linear regression model and Chi-square test were used to identify any significant association between the metabolic syndrome and NAFLD. Multiple logistic regression was used to analyze the associations between base characteristic of the study group (age, gender, mean blood pressure, fasting blood sugar total cholesterol, LDL-C, HDL-C, triglycerides, and waist circumference) and control group.

\section{RESULT}

Out of the 100 patients in the study group, 62 were females (62\%) and 38 were males (38\%). In control group out of the 50 patients, 29 (58\%) were females and $21(42 \%)$ were males. The statistical analysis showing the difference between baseline characteristics of the two groups is given in Table 1. Both the groups were similar with respect to age and sex distribution to avoid maximum biasing. In the study group, 67 patients (67\%) were having Grade I fatty liver, 34 (34\%) were having Grade II fatty liver, six $(6 \%)$ were having hepatomegaly with coarse echotexture (without any specification), and four (4\%) had cholelithiasis. However, fasting blood sugar, mean systolic pressure, diastolic pressure, BMI, waist circumference, total cholesterol, LDL-C, and triglycerides were significantly higher in the study group as compared to the control group. HDL-C was found to be significantly lower in the study group.

The linear regression, assessing the correlation between the components of metabolic syndrome with the severity of NFLDS in terms of grading showed significant positive linear association with total cholesterol, triglycerides, LDL-C, FBS and systolic blood pressure and negative linear association with HDL. In the study group, 67 patients (67\%) were having Grade I fatty liver, 34 patients (34\%) were having Grade II fatty liver, six patients $(6 \%)$ were having hepatomegaly with coarse echotexture (without any specification) and four patients (4\%) had cholelithiasis. NAFLD had a significant association with metabolic syndrome as assessed by Chi-square test $(\mathrm{p}=0.001)$ (Table 2$)$.

Logistic regression analysis showed significant association of NAFLD with LDL-C, triglyceride, and total cholesterol ( $\mathrm{p}=0.000$, CI-0.321-0.798) (Table 3).
Table 1: Baseline characteristics of the study population

\begin{tabular}{llll}
\hline $\begin{array}{l}\text { Baseline } \\
\text { characteristic }\end{array}$ & $\begin{array}{l}\text { Metabolic } \\
\text { syndrome/study } \\
\text { group (n=100) }\end{array}$ & $\begin{array}{l}\text { Control } \\
\text { group } \\
\text { (n=50) }\end{array}$ & p value \\
\hline Age & $48.60 \pm 3.96$ & $47.20 \pm 4.45$ & 0.360 \\
BMI & $28.90 \pm 3.4$ & $19.51 \pm 1.98$ & 0.000 \\
Waist circumference & $88.90 \pm 4.2$ & $85.34 \pm 4.23$ & 0.001 \\
FBS & $130.63 \pm 28.21$ & $87.02 \pm 8.76$ & 0.001 \\
SBP (mmHg) & $140 \pm 20.2$ & $119.82 \pm 9.98$ & 0.001 \\
DBP (mmHg) & $88.72 \pm 8.76$ & $76.20 \pm 5.64$ & 0.000 \\
Total & $215.76 \pm 25.89$ & $180.32 \pm 12.87$ & 0.001 \\
cholesterol (mg/dl) & & & \\
LDL-C (mg/dl) & $116.56 \pm 24.45$ & $86.50 \pm 18.59$ & 0.001 \\
HDL-C (mg/dl) & $42.34 \pm 5.64$ & $48.87 \pm 8.92$ & 0.005 \\
Triglycerides & $176.12 \pm 20.65$ & $130.45 \pm 16.78$ & 0.001 \\
NAFLD Grade I & $67.00 \pm 5.67$ & $4.00 \pm 2.45$ & 0.001 \\
NAFLD Grade II & $34.00 \pm 4.56$ & 0.00 & 0.001 \\
\hline
\end{tabular}

*BMI: Body mass index, FBS: Fasting blood sugar, LDL-C: Low-density lipoprotein cholesterol, HDL: High-density lipoprotein cholesterol, data are expressed in mean \pm standard deviation and $\mathrm{p}<0.005$ is reported statistical significant, SBP: Systolic blood pressure, DBP: Diastolic blood pressure

Table 2: Correlation between components of metabolic syndrome and non alcoholic fatty liver disease severity (using linear regression model)

\begin{tabular}{lll}
\hline Baseline characteristic & NAFLD & \\
\cline { 2 - 3 } & B & Significant \\
\hline FBS & 0.178 & 0.032 \\
SBP & 0.234 & 0.004 \\
DBP & 0.75 & 0.387 \\
TC & 0.421 & 0.000 \\
LDL-C & 0.542 & 0.000 \\
HDL-C & -0.243 & 0.001 \\
TG & 0.321 & 0.003 \\
WC & 0.056 & 0.232 \\
\hline
\end{tabular}

*BMI: Body mass index, FBS: Fasting blood sugar, SBP: Systolic blood pressure, DBP: Diastolic blood pressure, LDL-C: Low-density lipoprotein cholesterol, HDL: High density lipoprotein cholesterol, $\beta$ : Standardized linear regression coefficient

Table 3: Association between baseline characteristics and NAFLD in study group and control group (logistic regression analysis)

\begin{tabular}{lllll}
\hline $\begin{array}{l}\text { Baseline } \\
\text { characteristic }\end{array}$ & Odds ratio & \multicolumn{2}{l}{$\begin{array}{l}\mathbf{9 5 \%} \text { CI for odds } \\
\text { ratio }\end{array}$} & p value \\
\cline { 2 - 3 } & & Lower & Upper & \\
\hline Age & 1.958 & 1.156 & 3.314 & 0.012 \\
BMI & 1.310 & 0.670 & 2.562 & 0.429 \\
FBS & 1.328 & 0.860 & 2.050 & 0.200 \\
SBP & 1.714 & 1.128 & 2.603 & 0.011 \\
DBP & 1.707 & 1.081 & 2.697 & 0.021 \\
TC & 1.674 & 1.130 & 2.480 & 0.010 \\
LDL-C & 1.482 & 0.955 & 2.300 & 0.078 \\
HDL-C & 2.285 & 1.338 & 3.904 & 0.002 \\
TG & 1.666 & 1.125 & 2.468 & 0.010 \\
WC & 1.910 & 1.216 & 2.999 & 0.004 \\
NAFLD & 0.1194 & 0.041 & 0.346 & 0.000 \\
\hline
\end{tabular}

*BMI: Body mass index, FBS: Fasting blood sugar, SBP: Systolic blood pressure, DBP: Diastolic blood pressure, LDL-C: Low-density lipoprotein cholesterol, HDL: High-density lipoprotein cholesterol, $\beta$ : Standardized linear regression coefficient 


\section{DISCUSSION}

This is a hospital-based cross-sectional study conducted in Outpatient Department of Kayachikitsa, All India Institute of Ayurveda, New Delhi. The hospital is situated at the heart of city and represent general population at large in general practice. The study group included 100 patients with 62 females and 38 male. Control group included 50 patients with 29 females and 21 males. Both the groups were similar in the distribution of age and gender. In the study group, BMI, waist circumference, fasting blood sugar, systolic and diastolic blood pressure, total cholesterol, LDL-C, and triglycerides were significantly higher than the control group, whereas HDL was significantly lower comparative to control group. NAFLD was found to be significantly associated with the metabolic syndrome, in control group only four patients were reported for having Grade I fatty liver with slightly higher level of LDL-C. Linear regression assessment showed a significant positive association of NAFLD with LDL-C, triglycerides and total cholesterol and negative association with HDL-C. The insignificant finding of NAFLD in control group suggests that metabolic syndrome is preceded by metabolic syndrome or it can be said that NAFLDS is probably the first clinical stage of disturb metabolic functions. Previous researches have also critically reviewed a possible relationship between NAFLD and metabolic syndrome, but clinical data required for establishing the strength of association is missing. Thus, this clinical study was planned with the aim to analyze the relation with the two clinical entities and their effect on treatment modality. There are many research studies that have shown the relation of NAFLD with the component of metabolic syndrome, viz., study conducted by Portillo Sanchez et al. showed that prevalence of NAFLD (50\% and $56 \%$ of NAFLD patients, respectively) in overweight/obese patients with Type 2 diabetes mellitus and having normal aminotransferases is much higher than previously reported [13]. They also reported that higher plasma A1c was associated with greater prevalence of NAFLD and accumulation of liver triglycerides $(\mathrm{p}=0.01)$. Patients with NAFLD had severe systemic (liver/muscle) and particularly adipose tissues insulin resistance $(\mathrm{p}<0.01)$. Bhatt and Smith had reported that NAFLD is highly prevent in Type 2 diabetes as well as Type 1 . In Type 2 diabetes, its incidence is directly related to insulin resistance and obesity [14]. They suggested that weight reduction might prove helpful in reversing the pathology of NAFLD in Type 2 diabetes. A position paper published by the combined effort of Indian National Association for study of the liver, Endocrine Society of India, Indian College of Cardiology and Indian Society of Gastroenetrology gives the detail description about the association of NAFLD and metabolic syndrome in Indian population and recommended a multidisciplinary approach involving hepatologist, internists, cardiologist and also endocrinologists for the proper management of NAFLD [15]. Diehl in his article emphasizes the impact of fatty liver disease on hepatic complications such as fibrosis, cirrhosis, and even carcinoma [16]. He says that fatty liver disease should not be taken innocuous condition as previously assumed and give the evidence for its association with other morbid diseases such as metabolic syndrome and hypertension. The authors of the article carried out a survey study on selected subgroup of hypertensives who were having no risk factors for hepatic steatosis and reported that despite excluding all possible risk factors for fatty liver or hepatic steatosis $30 \%$ of the hypertensive were having fatty liver disease. He, further concluded that the prevalence of fatty liver disease in nonobese, nondiabetic hypertensive adults is almost two times to the historical prevalence of fatty liver in general population and almost three times to the age and sex matched group of parallel control. Zhang and Lu mentioned that at least $20-80 \%$ of NAFLD patients are having dyslipidemia (high level of triglycerides, LDL and low level of HDL) that increases the risk of cardiovascular complications in such patients [17]. They suggest that there exists a close relationship between Type 2 diabetes, obesity, metabolic syndrome and NAFLD. NASH significantly elevate the level of oxidized LDL-C. The characteristic feature of dyslipidemia in NAFLD is hypertriglyceridemia, low HDL-C and high LDL-C levels, which is atherogenic dyslipidemia. It is wellknown fact that dyslipidemia causes insulin resistance which in turn increases the free fatty acid (FFA) flux. The increased FFA level raises triglyceride and VLDL production and triggers oxidative stress and lipid peroxidation that deteriorates further the functioning of hepatocytes causing NAFLD and setting a vicious cycle [18]. Thus, it is evident from the above discussion that there is an established link between NAFLD and all the components of metabolic syndrome, i.e., hypertension, Type 2 diabetes, dyslipidemia and obesity and this clinical trial justified it by providing clinical data.

\section{CONCLUSION}

There exist a significant association between NFLDS and metabolic syndrome. This clinical trial showed that NAFLD relation with metabolic syndrome but further extensive studies is required to establish the cause and consequence relation between the two. The occurrence of fatty liver disease in control group suggest that it may be the first clinical sign signifying metabolic disturbance and necessitate the life style modulation and hypolipidemic drugs to prevent progression as metabolic syndrome.

\section{ACKNOWLEDGMENT}

The author is thankful to Dr. Nasreen Ahmed (PG Scholar, Department of Kayachikitsa, AIIA, New Delhi) for her support in collection of data.

\section{REFERENCES}

1. Huang PL. A comprehensive definition for metabolic syndrome. Dis Model Mech 2009;2(5-6):231-7.

2. Abd El-Kader SM, El-Den Ashmawy EM. Non-alcoholic fatty liver disease: The diagnosis and management. World $\mathrm{J}$ Hepatol 2015;7(6):846-58

3. Ahmed HH, Salem AM, Mohamed MR, Shahat AA, Khalil WK, Mohamed SH. Experimental evidences for the promising therapeutic role of Vitis vinifera seed extract against non alcoholic steatohepatitis. Int J Pharm Pharm Sci 2015;7(2):417-24

4. Khandelwal VK, Singh P, Ravingerova T, Chaudhary M. Evaluation of ezenus in an experimental model of diet-induced alcoholic and non-alcoholic fatty liver condition in rats. Int J Pharm Pharm Sci 2015;7(5):247-52

5. Fabbrini E, Sullivan S, Klein S. Obesity and nonalcoholic fatty liver disease: Biochemical, metabolic, and clinical implications. Hepatology 2010;51(2):679-89.

6. Vuppalanchi R, Chalasani N. Non-alcoholic fatty liver disease and nonalcoholic steatohepatitis: Selected practical issues in their evaluation and management. Hepatology 2009;49(1):306-17.

7. Birkenfeld AL, Shulman GI. Non alcoholic fatty liver disease, hepatic insulin resistance and Type 2 diabetes. Hepatology 2014:59(2):713-23.

8. Paschos P, Paletas K. Non alcoholic fatty liver disease and metabolic syndrome. Hippokratia 2009;13(1): 9-19.

9. Sundaram SS, Zeitler P, Nadeau K. The Metabolic syndrome and non-alcoholic fatty liver disease in children. Curr Opin Pediatrics 2009;21(4):529-35.

10. Diehl AM. Fatty liver, hypertension and the metabolic syndrome. Gut 2004;53923-24.

11. Rahman M, Berenson AB. Accuracy of current body mass index obesity classification for white, black and Hispanic reproductive-age women. Obstet Gy necol 2010;115(5):982-8.

12. Aziz N, Kallur SD, Nirmalan PK. Implications of the revised consensus body mass indices for Asian Indians on clinical obstetric practice. J Clin Diagn Res 2014;8(5):OC01-3.

13. Portillo-Sanchez P, Bril F, Maximos M, Lomonaco R, Biernacki D, Orsak B, et al. High Prevalence of nonalcoholic fatty liver disease in patients with Type 2 diabetes mellitus and normal plasma aminotransferase levels. J Clin Endocrinol Metab 2015;100(6):2231-8.

14. Bhatt HB, Smith RJ. Fatty liver disease in diabetes mellitus. Hepatobiliary Surg Nutr 2015;4(2):101-8

15. Duseja A, Singh SP, Saraswat VA, Acharya SK, Chawla YK, Chowdhury S, et al. Non-alcoholic fatty liver disease and metabolic syndrome-position paper of the Indian national association for the study of the liver, endocrine society of india, indian college of cardiology and Indian society of gastroenterology. J Clin Exp Hepatol 2015;5(1):51-68.

16. Diehl AM. Fatty liver, hypertension, and the metabolic syndrome. Gut 2004;53(7):923-4.

17. Zhang QQ, Lu LG. Nonalcoholic fatty liver disease: Dyslipidemia, risk for cardiovascular complications, and treatment strategy. J Clin Transl Hepatol 2015;3(1):78-84.

18. Sears B, Perry M. The role of fatty acids in insulin resistance. Lipids Health Dis 2015;14:121. 